\title{
AVALIAÇÃO DO TESTE DE AGLUTINAÇÃO DIRETA NA DETECÇÃO DA INFECÇÃO POR LEISHMANIA (VIANNIA) BRAZILIENSIS EM POSSÍVEIS RESERVATÓRIOS DE LEISHMANIOSE TEGUMENTAR AMERICANA NO ESTADO DO CEARÁ
}

\section{Haroldo Sérgio da S. Bezerra, Joseval da Rocha Viana, Maria Jania Texeira, Cristina Sousa Chaves, Daniela Bastos de Araújo e José Hermênio C. Lima Filho}

\begin{abstract}
Com o objetivo de avaliar a aplicabilidade do Teste de Aglutinação Direta (TAD) como método de deteç̧ão da infecção natural canina por Leishmania (Viannia) braziliensis, foi realizado um estudo envolvendo soros de cães residentes em uma área endêmica de leishmaniose tegumentar, Serra de Baturité, $C E$ e soros de câes provenientes da cidade de Curitiba, $P R$, área não endêmica de leishmaniose. Os resultados obtidos com o TAD neste trabalho indican a possibilidade do uso deste teste sorológico para levantanentos epidemiólogicos da infecção em reservatórios; neste estudo, o cão doméstico.
\end{abstract}

Palavras-chaves: Teste de aglutinação direta. Leishmania (V.) braziliensis. Leishmaniose tegumentar. Cão.

Leishmaniose tegumentaré uma doença causada por várias espécies de protozoários pertencentes ao gênero Leishmania. Estes parasitas podem ocorrer sob duas formas evolutivas: as formas flageladas denominadas de promastigotas, encontradas no trato digestivo dos flebotomíneos vetores, e as formas amastigotas encontradas parasitando macrófagos dos hospedeiros mamíferos ${ }^{1}$,

No Estado do Ceará, a leishmaniose tegumentar representa umimportante problema de saúde pública. De acordo com as estatísticas do Ministério da Saúde, o Ceará é o estado da região Nordeste onde mais ocorrem casos de leishmaniose tegumentar $(44,6 \%)$ e leishmaniose visceral (68\%). A principal zona endêmica de leishmaniose tegumentar do Ceará está localizada na Serra de Baturité ${ }^{2}$, uma área não endêmica para leishmaniose visceral e doença de Chagas, que dista aproximadamente $100 \mathrm{~km} \mathrm{da}$ capital, Fortaleza. Vários estudos sobre a epidemiologia desta doença na área de Baturité vêm

\footnotetext{
Núcleo de Medicina Tropical/Departamento de Patologia e Medicina Legal da Universidade Federal do Ceará, Fortaleza, CE.

Suporte Financeiro: FNS/PCDEN, World Bank.

Endereço para correspondência: Dr. Haroldo Sérgio da Silva Bezerra. Núcleo de Medicina Tropical/UFCE. R. Alexandre Baraúna 949, Rodolfo Teófilo, CP: 3166, 60431-970 Fortaleza, CE.

Recebido para publicação em 08/01/96
}

sendo realizados pelo Núcleo de Medicina Tropical/ DPML/UFC. Tais estudos têm auxiliado a desvendar importantes pontos sobre o ciclo de transmissão da leishmaniose tegumentar em Baturité ${ }^{2}{ }^{4}$. O agente etiológico nesta área foi caracterizado como Leishmania (V.) braziliensis ${ }^{2}$. Quanto aos reservatórios silvestres, pouco se sabe sobre eles, porém estudos de Oliveira Lima (dados não publicados) têm apontado um papel importante do cão no ciclo de transmissão doméstica desta endemia na área de Baturité. Em outras regiões endêmicas de leishmaniose tegumentar, diversos estudos têm também apontado o cão como importante reservatório doméstico desta doença ${ }^{56}$.

Neste trabalho, apresentamos uma avaliação da aplicabilidade do Teste de Aglutinação Direta (TAD) como método de deteç̧ão da infecção natural canina por Leishmania (V.) braziliensis, utilizando soros de cães residentes na Serra de Baturité, importante zona endêmica desta doença no Ceará.

\section{MATERIAL E MÉTODOS}

\section{Soros}

Os soros utilizados fazem parte do banco de soros do Núcleo de Medicina Tropical/UFC, sendo estes divididos em 2 grupos. O primeiro grupo, 
Bezerra HSS, Viana JR, Teixeira MJ, Chaves CS, Araújo DB, Lima Filho JHC. Avaliação do teste de aglutinação direta na deteç̧ão da infecção por Leishmania (Viannia) braziliensis em possiveis reservatórios de leishmaniose tegumentar americana no Estado do Ceará. Revista da Sociedade Brasileira de Medicina Tropical 29:181-184, mar-abr, 1996.

consistiu de 40 amostras de soros de cães (de diferentes sexos, idades e raças) residentes na Serra de Baturité, 20 destes infectados por Leishmania (V.) braziliensis, com confirmação parasitológica através de biópsias e culturas em meio NNN. de material de pele, figado ou baço. Todas as cepas foram caracterizadas através da Técnica de Eletroforese de Isoenzimas?; os outros 20 tinham resultado positivo para a técnica de imunofluorescência indireta utilizando antígeno de Leishmania (V.) braziliensis ${ }^{8}$. O segundo grupo, constou de 18 amostras de soros de cães (de diferentes sexos, idades e raças) provenientes da cidade de Curitiba (PR), área não endêmica de leishmaniose, gentilmente cedidos ao Núcleo de Medicina Tropical pelo Dr. Natal Jatair Camargo. Esses animais não apresentaram qualquer sinal clínico de leishmaniose, bem como soro não reagente frente a antígenos de L. chagasi através da Técnica de ELISA ${ }^{9}$ e Imunofluorescência indireta para Leishmania braziliensis.

\section{Preparação de antígeno}

Promastigotas de Leishmania (Viannia) braziliensis (MCAN/BR/92/19914) foram cultivadas a $26^{\circ} \mathrm{C}$ em meio Shneider (Sigma) contendo $20 \%$ de soro bovino fetal inativado (SBF), $2 \%$ de urina humana e antibióticos (penicilina, $200 \mathrm{U} / \mathrm{ml}$; streptomicina, $200 \mu \mathrm{g} / \mathrm{ml}$ e gentamicina, $1 \mu \mathrm{g} / \mathrm{ml}$ ), e utilizadas na produção de antígenos segundo o método de Harith e cols ${ }^{10}$; os parasitas foram lavados em solução de Locke $(\mathrm{pH} 7,7)$ a $4{ }^{\circ} \mathrm{C}$, tratados com tripsina (1:250, Gibco, Detroit, USA) a $0,4 \%$ em solução de Locke por $45 \mathrm{~min}$ a $37^{\circ} \mathrm{C}$ e fixados por $20 \mathrm{~h} \mathrm{a} 4^{\circ} \mathrm{C}$ com formalina a $1 \% \mathrm{em}$ solução de Locke. Após lavagem em salina-citrato $(0,15 \mathrm{M}$ cloreto de sódio $+0,056 \mathrm{M}$ citrato de sódio, $\mathrm{pH} 7,4$ ), os parasitas foram corados com azu! brilhante de Coomassie a $0,1 \%$ em salina por $90 \mathrm{~min}$, sob agitação. Parasitas corados foram lavados em salina-citrato $(0,15 \mathrm{M}$ cloreto de sódio $+0,056 \mathrm{M}$ citrato de sódio, $\mathrm{pH} 7,4)$ e ressuspendidos com formalina a $1 \%$ em salina-citrato $(0,15 \mathrm{M}$ cloreto de sódio $+0,056 \mathrm{M}$ citrato de sódio, $\mathrm{pH} 7,4$ ). A suspensão foi filtrada em filtro de poros de $30 \mu \mathrm{m}$ e a concentração de parasitas foi ajustada para $2 \times 10^{7} \mathrm{ml}$. A preparação foi mantida no escuro a $4^{\circ} \mathrm{C}$ até sua utilização.

\section{aste de Aglutinação Direta}

Para a realização do teste, diluições seriadas de $1: 10$ até $1: 20,480$ dos soros foram feitas com $\mathrm{NaCl}$ a $0,9 \%$ suplementado com $0,2 \%$ de gelatina e 2mercaptoetanol $0,1 \mathrm{M}$ (Merck), perfazendo um volume final de $50 \mu \mathrm{l} /$ poço, começando da segunda coluna em uma microplaca com poços de fundo em "V". A primeira coluna, contendo somente diluente, foi usada como controle. Após a incubação da placa durante $1 \mathrm{~h}$ a $37^{\circ} \mathrm{C}$, foi adicionado igual volume de antígeno $(50 \mu \mathrm{l}) \mathrm{em}$ cada poço. As placas foram então incubadas durante $18 \mathrm{~h}$ a $26^{\circ} \mathrm{C}$ e lidas em seguida. O título de aglutinação foi determinado como sendo o do poço anterior àquele onde se observava um "botão" idêntico ao observado no poço controle.

\section{RESULTADOS}

Um total de 58 soros de cães de diferentes raças, idades e sexos foi utilizado nos testes. Dos 40 soros positivos para outras técnicas, 34 (85\%) demonstraram títulos $\geq 1: 160$ chegando até a 1:2560, e somente $2(5 \%)$ apresentaram títulos $\leq 1: 20$. Dos soros restantes, negativos, apenas $2(11,1 \%)$ apresentaram títulos iguais a 1:160, sendo esta também sua maior titulação, e todos os outros $(88,9 \%)$ tiveram títulos menores que 1:160 (Tabela 1). A distribuição dos soros em relação aos seus títulos e percentuais estão demonstrados na Figura 1.

Tabela 1 - Títulos do teste de aglutinação direta de uma amostra de 58 soros de cães usando antigenode Leismania (V.) brasiliensis.

\begin{tabular}{lcccc}
\hline Diagnóstico & \multicolumn{4}{c}{ Títulos } \\
\cline { 2 - 5 } & $\leq 1: 20$ & $1: 40$ & $1: 80$ & $\geq 1: 60$ \\
\hline Positivos & 2 & 1 & 3 & 34 \\
Negativos & 8 & 5 & 3 & 2 \\
\hline
\end{tabular}


Bezerra HSS, Viana JR, Teixeira MJ, Chaves CS, Araújo DB, Lima Filho JHC. Avaliação do teste de aglutinação direta na deteç̧ão da infecção por Leishmania (Viannia) braziliensis em possiveis reservatórios de leishmaniose tegumentar americana no Estado do Ceará. Revista da Sociedade Brasileira de Medicina Tropical 29:181-184, mar-abr, 1996.

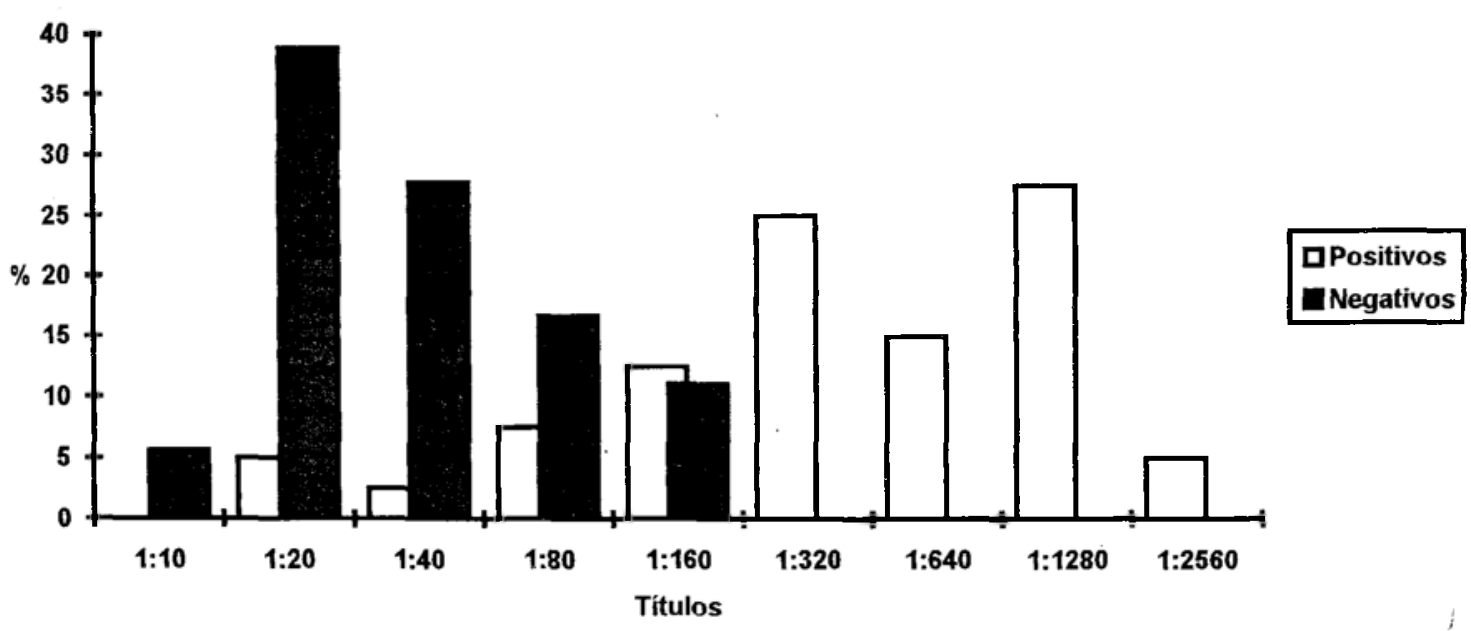

Figura 1 - Distribuição dos percentuais e titules do teste de aglutinação direta dé uma amostra de 58 cães com diagnóstico positivos e negativos para Leismania (V.) braziliensis.

\section{DISCUSSÃO}

O diagnóstico de infecção por Leishmania em cães, nas zonas endêmicas, tem sido feito por demonstração do parasito e/ou técnicas sorológicas. Os resultados obtidos com o TAD neste estudo indicam a possibilidade do uso deste teste sorológico para o levantamento epidemiológico da infeç̧ão em reservatórios (no caso o cão). Um cut-off de 1:160 foi capaz de discriminar $85 \%$ dos cães infectados; neste nível ainda, somente $11 \%$ de falso-positivos foram detectados.

Como demonstrado neste estudo, o TAD por sua facilidade de realização, bem como baixo custo, poderá ser usado na investigação da infecção por Leishmania para determinação de reservatórios da leishmaniose tegumentar, principalmente nas regiões onde se possa excluir leishmaniose visceral edoença de Chagas. Estudos preliminares estão sendo realizados neste laboratório para evolução desta pesquisa, visando estudar, inclusive, animais silvestres.

\section{SUMMARY}

In order to evaluate the aplicability of the Direct Agglutination Test (DAT) as a method of detection of the natural canine infection for Leishmania (Viannia) braziliensis, it was realized a study involving the serum of naive dogs from Serra de Baturite, Ceara State, an endemic area of cutaneous leishmaniasis, and others from Curitiba, Parana State, a non endemic area. The results indicate the possibility of using this sorologic test to the epidemiologic research of the infection in the reservoir host; in this case, the dog.

Key-words: Direct Agglutination Test. Leishmania (V.) braziliensis. Cutaneous leishmaniasis. Dog.

\section{REFERÊNCIAS BILIOGRÁFICAS}

1. Pearson RD, Wilson ME. Host Defenses Against Prototypical Intracellular Protozoans, the Leishmania. In Parasitic Infections in the Compromised Host (Peter D. Walzer and Robert M. Genta, eds.). Marcel Dekker. Ine.; New York and Basel, Chap. 2, p.31-81, 1989.

2. Vasconcelos IAB, Vasconcelos AW, Momem H, Grimaldi Jr. G, Alencar JE. Epidemiological Studies on American Leishmaniasis in Ceará State, Brazil. Molecular Characterization of Leishmania Isolate. American Tropical
Medicine and Parasitology 82:547-554, 1988.

3. Queiroz RG, Vasconcelos IAB, Vasconcelos AW, Pessoa FAC, Sousa RN, David JR. Cutaneous Leishmaniasis in Northeast Brazil: Incrimination of Lutzomyia whitmani (Diptera: Psycodidae) as a Vector of Leishmania braziliensis in Baturité Municipality. The American Journal of Tropical Medicine and Hygiene, 1994.

4. Azevedo ACR, Rangel EF, Queiroz RG. Lutzomyia migonei (França, 1920) Naturally Infected with Peripylarian 
Bezerra HSS, Viana JR, Teixeira MJ, Chaves CS, Araújo DB, Lima Filho JHC. Avaliação do teste de aglutinação direta na deteç̧ão da infeç̧ão por Leishmania (Viannia) braziliensis em possiveis reservatórios de leishmaniose tegumentar americana no Estado do Ceará. Revista da Sociedade Brasileira de Medicina Tropical 29:181-184, mar-abr, 1996.

flagellates in Baturité, a Focus of Cutaneous Leishmaniasis in Ceará State, Brazil. Memórias do Instituto Oswaldo Cruz 85:479, 1990.

5. Pirmez C, Coutinho SG, Marzochi MCA, Nunes MP, Grimaldi Jr. G. Canine American Cutaneous Leishmaniasis: a Clinical and Immunological Study in Dogs Naturally Infected with Leishmania braziliensis in an Endemic Area of Rio de Janeiro, Brazil. The American Journal of Tropical Medicine and Hygiene 38:52-58, 1988.

6. Falqueto A, Coura JR, Barros GC, Grimaldi Fo.G, Sessa PA, Carias VRD, de Jesus AC, de Alencar, JTA. Participação do Cão no Ciclo de Transmissão da Leishmaniose Tegumentar no Município de Viana, Estado do Espírito Santo, Brasil. Memórias do Instituto Oswaldo Cruz 81:155-163, 1986.

7. Momen H, Grimaldi Jr. G, Pacheco RS, Jaffe CL, McMahon-Pratt D, Marzochi MCA. Brazilian Leishmania
Stocks Phenotypically Similar to Leishmania major. The American Journal of Tropical Medicine and Hygiene 34:1076-1084, 1985 .

8. Bittencourt AL, Sodre A, Andrade ZA. Pesquisa de Anticorpos Circulantes pelo Método de Imunofluorescência na Leishmaniose Tegumentar. Revista do Instituto de Medicina Tropical de São Paulo 10:247-252, 1968.

9. Evans TG, Vasconcelos IAB, Lima JW, Teixeira MJ, McAullife IT, Lopes UG, Pearson RD, Vasconcelos AW. Canine Visceral Leishmaniasis in Northeast Brazil: Assessment of Serodiagnostic Methods. The American Journal of Tropical Medicine and Hygiene 42:118-123. 1990.

10. Harith EA, et al. Improvement of a Direct Aglutination Test for Field Studies of Visceral Leishmaniasis. Journal of Clinical Microbiology 26:1321-1325, 1988. 